\title{
Stem cell fate outcomes modulated by the CDK inhibitors
}

\author{
Tao Cheng ${ }^{1}$ \\ ${ }^{1}$ University of Pittsburgh Cancer Institute and Institute of Hematology \& Hospital for Blood Diseases, Chinese Academy of \\ Medical Sciences \& Peking Union Medical College
}

Efficacy of stem cell therapy is highly dependent upon our ability to manipulate the mechanisms of selfrenewal. Cell cycle is a fundamental aspect underlying stem cell self-renewal. But cell cycle progression per sea does not necessarily yield the self-renewal outcome because a daughter stem cell faces the choices of self-renewal, differentiation and apoptosis. Hence, lifting cell cycle brakes in stem cells may have different outcomes depending on a specific context. Increasing evidence suggests that the cyclin-dependent kinase inhibitors (CKIs) such as p21Cip1/ Waf1, p27kip1, p16iNK4A, and p18INK4C (p21, p27, p16 and p18 hereafter) are involved in stem cell regulation, as mainly demonstrated with murine hematopoietic stem cells (HSCs) (Cheng, 2004). In particular, we have shown a significant increase of HSC self-renewal in the absence of p18 (Yuan et al., 2004) and such an effect could last over 3 years in serial bone marrow transplant model (Yuan et al., 2006). But the actual roles of these CKIs in HSCs appear to be distinct as p21 and p18 have opposite effects (Cheng et al., 2000b; Yu et al., 2006) whereas p16 seems to have a more specific effect on HSC aging (Janzen et al., 2006). Like p18, however, p27 was also reported to inhibit HSC selfrenewal based on the fact that the competitive repopulating units were increased in p27-/- mouse bone marrow (Walkley et al., 2005) in contrast to the result in a previous report (Cheng et al., 2000a). To further gauge the impact of p18 versus p27 on HSCs, we have directly compared the long-term repopulating ability of p18-/- and p27-/hematopoietic cells with different congenic strains and our results demonstrated a striking advantage of p18-null over p27-null during the long-term engraftment, preferentially in the HSC compartment. In short, our series studies suggest a dominant role of p18-mediated signaling in self-renewal of murine HSC though its molecular network is yet to be defined.

Cell Research (2008) 18:s94. doi: 10.1038/cr.2008.184; published online 4 August 2008

Correspondence: Tao Cheng

E-mail: Chengt@upmc.edu

Dr Tao Cheng is tenured Associate Professor of Radiation Oncology and Director of Stem Cell Biology at University of Pittsburgh Cancer Institute. Prior to his current tenure, he was Assistant Professor of Medicine at Harvard Medical School. He is also holding an adjunct Professorship at
Chinese Academy of Medical Sciences and Peking Union Medical College. Dr Cheng's work on stem cell research has been published in many highprofile journals including Science, Nature and other Nature sister journals. He was a recipient of multiple awards such as Young Faculty Scholar Award from the American Society of Hematology (2002) and Chang-Jiang Scholarship from the Ministry of Education of China (2007). 